\title{
Hand And Foot Dermatoses In Dermatology Outpatients
}

\author{
G Demirci, I Altunay, B Ates, G Gokdemir
}

\section{Citation}

G Demirci, I Altunay, B Ates, G Gokdemir. Hand And Foot Dermatoses In Dermatology Outpatients. The Internet Journal of Dermatology. 2010 Volume 8 Number 2.

DOI: $\underline{10.5580 / 150 \mathrm{e}}$

\begin{abstract}
Aims: We aimed to sort out clinical types, localizations and frequencies of only hand dermatoses (HD), only foot dermatoses (FD) and both hand and foot dermatoses (HFD) and to evaluate the relationship with sociodemographic parameters. Methods and Material: Dermatological examination of 450 outpatients was done and the relationship between the frequencies of diseases, localizations of the lesions and sociodemographic features was assessed statistically. Results: The frequencies of dermatoses were $34 \%$ for tinea pedis, $29 \%$ for eczema, $9 \%$ for warts, $5 \%$ for corns, $3 \%$ for psoriasis and $21 \%$ for the others in the participiants. Tinea pedis was the most frequent dermatose of men while eczema of women. HD except warts were the most frequent in housewives while warts in students. The most frequent HFD were psoriasis, eczema and tinea.Statistical analysis used: Chi square test, one way ANOVA test and tukey's test were used for statistical assessments Results: The frequencies of dermatoses were $34 \%$ for tinea pedis, $29 \%$ for eczema, $9 \%$ for warts, $5 \%$ for corns, $3 \%$ for psoriasis and $21 \%$ for the others in the participants. Tinea pedis was the most frequent dermatose of men while eczema of women. HD except warts were the most frequent in housewives while warts in students. The most frequent HFD were psoriasis, eczema and tinea.Conclusions: We believe that elucidating the frequencies, clinical localizations and demographic features of these dermatoses will be helpful both for diagnosing and decreasing the incidence of these diseases in general population which seem to be a common health problem.
\end{abstract}

\section{INTRODUCTION}

Hand and foot dermatoses constitute one group of the most frequent seen diseases in daily dermatological practice.

Hands are the most active organs which keep us in contact with our environment. Thus, dermatoses involving the hands affect their functionality in daily routine life to some extent. They are also mostly in contact with microorganisms. Infections on the hands spread around more quickly by hands. However, even in non-contagious hand dermatoses (HD), patients' social environment usually have a prejudice as those dermatoses are non hygienic and contagious and they avoid having contact with these patients just because of their appearance. HD which can be easily recognised by other people affect patients in especially psychosocial domain. HD may have negative effects on both communication with other people and sexual contact so all of them make patient embarrassed in his social and business life with many restrictions. ${ }^{1,2}$

While visible character and effects on functionality of HD are apparent, foot dermatoses (FD) which are covered by socks and shoes most of the time and can not be seen by others, are not considered to be as a real disease by some patients. Beside these, if a patient does not tell the physician about the problem of his feet, the physician may forget to examine the feet in routine dermatological examination. In these cases diagnosis and treatment is done lately and the impairment of barrier of the foot skin predisposes to many other diseases such as erysipelas, lymphangitis, cellulitis to occur. Especially in summertime wearing sandals and revealing shoes make FD visible by others and this affect patients' psychosocial status negatively.

Some of the dermatoses start to appear first on the hand and foot (HF) and spread to whole body afterwards, while some start appearing first on and the body then spread to the extremities. Some of the hand and foot dermatoses (HFD) may be a sign of the systemic diseases ${ }^{3-6}$ and some are only specifically localized on the hands and feet. Infectious and allergic contact eczema due to detergents may be seen more common than general. $^{7-9}$

In this study our purpose was to find out the clinical types and frequencies of HF dermatoses, and relationships between age, sex and occupational parameters in patients 
who had only HD, only FD or both HFD.

\section{SUBJECTS AND METHODS}

450 dermatology outpatients, (194 men and 256 women, mean age: 38.1 ) participated into the study. The patients were collected from those applied to our outpatient clinic with only HD, only FD or with HFD in a period of 6 months. Sociodemographic features such as age, gender, occupation and clinical features were noted; histopathologic and laboratory investigations were done if necessary. Diagnoses were classified into six main groups as allergic reactions, inflammatory dermatoses, infectious skin diseases, tumoral diseases and reactions due to physical factors. Nail disorders were excluded in this assessment. The localizations of lesions for both the hands and feet were evaluated at seven categories including the dorsal and palmar surfaces of the hands and feet, the fingers and toes and mixed localizations. The relationship between the frequencies of diseases, localizations of the lesions on the hands or feet and sociodemographic features were assessed statistically. Chi square test, one way ANOVA test and tukey's test were used for statistical assessments and $\mathrm{p}<0.05$ was considered significant.

\section{RESULTS}

A total of 450 patients were included and the number of female patients $(256 ; 56.9 \%)$ were statistically more than the male patients $(194 ; 43.1 \%)$ in all of the group of dermatoses. $(\mathrm{p}<0,005)$ (Graphic 1).

The median age was $38 \pm 34.8$ years (age range $7-88$ ). Patients' age medians were statistically different in each dermatose group $(\mathrm{p}<0,005)$; in the group of tinea pedis $44 \pm 35$, in the group of eczema $32.9 \pm 28$, in the group of warts $24,8 \pm 27$, in the group of corns $35.4 \pm 31$, in the group of psoriasis $40.2 \pm 32.6$ and in the others $40.9 \pm 37.2$ respectively. The median age of the group of warts was smaller than the group of tinea, psoriasis and the others. The median age of the group of eczema was smaller than tinea pedis and others (Graphic 2).

The ratios of dermatoses diagnosed on the participants were $34 \%$ for tinea, $29 \%$ for eczema, $5 \%$ for warts, $5 \%$ for corns, $5 \%$ for psoriasis and $21 \%$ for the other dermatoses. The number of the patients with tinea pedis and eczema were more than the others (Graphic 3).

The median dermatose duration time of all participants was $27.9 \pm 95.2$ months. According to dermatose types the median duration time was not statistically different from each other $(p>0,005)$.

The ratio of FD $(235 ; 52.2 \%)$ was more than the ratio of HD (173; \%38) and HFD (42; 9.3\%).

In the group of eczema, hand localization $(99 ; 77 \%)$ was more frequent than foot localization $(21 ; 16.3 \%)$ and hand and foot localization $(9 ; 7 \%)$. In the group of warts, hand localization ratio $(28 ; 67 \%)$ was more than foot $(13 ; 31 \%)$ and foot and hand localization ratio $(1 ; 2.1 \%)$. In the group of psoriasis, the ratios of hand localization $(3 ; 23 \%)$, foot localization $(2 ; 15.4 \%)$ and hand and foot localization $(8$; $61.5 \%$ ) were not different from each other.

According to the localizations of dermatoses in women foot localization was $49,6 \%$, hand localization was $44.9 \%$ and hand and foot localization was $5.5 \%$. In men foot localization was $55.7 \%$, hand localization was $29.9 \%$, hand and foot localization was $14.4 \%$. Foot localisation was found statistically more common in men then women (Table 1).

Tinea $(151 ; \% 54)$ was the most seen dermatose in the foot localization and was most frequent dermatose found in men $(79 ; 40 \%)$. Corns $(36 ; 13 \%)$, eczema $(31 ; 11 \%)$ and warts $(14 ; 5 \%)$ are the other frequent dermatoses seen on foot and mostly localized on foot fingers $(110 ; 46,8 \%)$.

Eczema $(108 ; 51 \%)$ was the most seen dermatose on hand and in women. Warts $(30 ; 14 \%)$, psoriasis $(14 ; 7 \%)$ and paronychia $(7 ; 3 \%)$ were the other frequent dermatoses on hand localisation. The lesions were mostly localised on the dorsum of the hand $(64 ; 37,2 \%)$.

On hand and foot localization the most frequent dermatoses were as follows; psoriasis $(10 ; 23 \%)$, eczema $(9 ; 20 \%)$ and tinea $(8 ; 19 \%)$.

In occupational groups all the dermatoses except warts were seen most in housewives while warts were most frequent in students. There is not statistically difference between psoriasis and occupations. The most frequent occupation for all the dermatose group was housework (Tablo 2).

We needed histopathological examination for the diagnosis only of the patients. The most frequent dermatoses were psoriasis $(7 ; 12,9 \%)$, eczema $(6 ; 11,1 \%)$, bowen $(2 ; 3,7 \%)$ and erytema nodosum $(2 ; 3,7 \%)$. 


\section{Figure 1}

Graphic 1. Ratio of male and female patients in all group of dermatoses

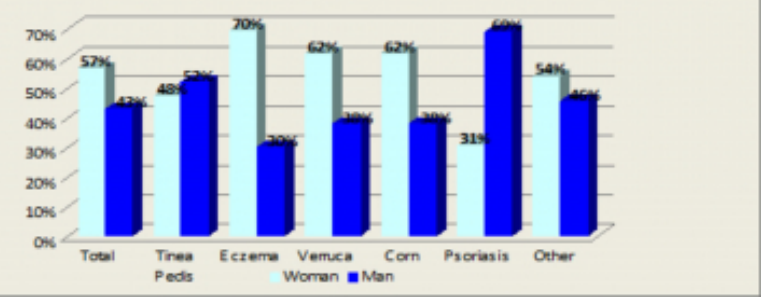

\section{Figure 2}

Graphic 2. The median age of patients in dermatose groups

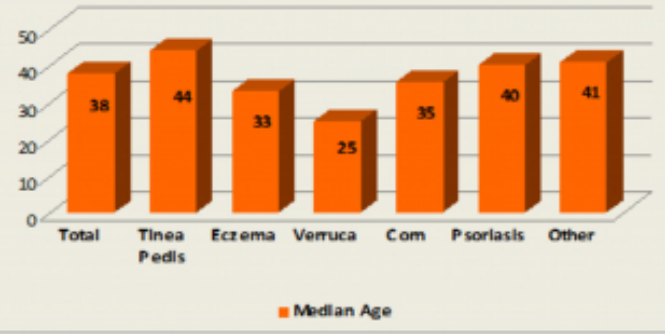

\section{Figure 3}

Graphic 3. The frequency of dermatoses in all of the patients

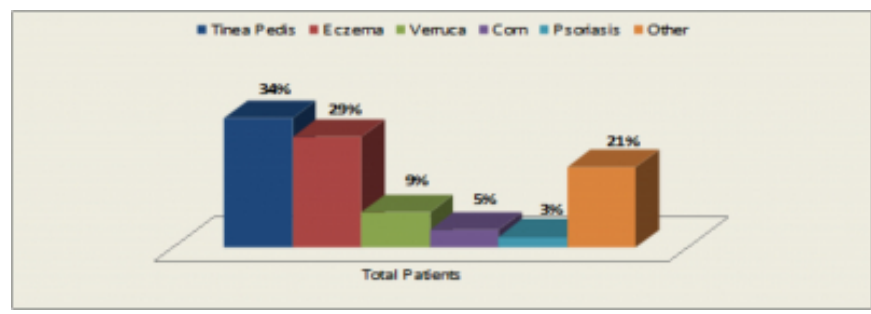

\section{Figure 4}

Table 1.Localizations of dermatoses in men and women

\begin{tabular}{|c|c|c|c|c|c|c|}
\hline & & \multicolumn{3}{|c|}{ Localization of Dermatoses } & \multirow[b]{2}{*}{ Tota! } & \multirow[b]{2}{*}{ p } \\
\hline & & Hand & Foot & Hand and Foot & & \\
\hline \multirow{2}{*}{ Woman } & n & 115 & 127 & 14 & 256 & \multirow{6}{*}{0,000} \\
\hline & $s$ & 44 क़ & 49,65 & $5,5 \%$ & $1000 \%$ & \\
\hline \multirow{2}{*}{ Man } & n & 58 & 108 & 28 & 194 & \\
\hline & s & 29,95 & $55,7 \%$ & 14,45 & 100,06 & \\
\hline \multirow[b]{2}{*}{ Total } & $\frac{n}{n}$ & $1 / 3$ & 235 & 42 & 450 & \\
\hline & $\$$ & 38.45 & $522 \%$ & 9.35 & $1000 \mathrm{~s}$ & \\
\hline
\end{tabular}

\section{Figure 5}

Table 2.The relationship between dermatoses and occupations

\begin{tabular}{|c|c|c|c|c|c|c|c|c|}
\hline \multicolumn{2}{|l|}{ Occupation } & $\begin{array}{l}\text { Timea } \\
\text { Pedis }\end{array}$ & Eczema & Verruca & Com & Psoriasis & Other & Total \\
\hline \multirow{2}{*}{ Housewife } & $\mathrm{n}$ & 52 & 54 & 13 & 9 & 4 & 36 & 170 \\
\hline & $\%$ & $36,4 \%$ & $41,9 \%$ & $31.0 \%$ & $42.9 \%$ & $30,8 \%$ & $40,4 \%$ & $37,8 \%$ \\
\hline \multirow{2}{*}{ Worker } & $n$ & 25 & 24 & 3 & 5 & 2 & 16 & 75 \\
\hline & $\%$ & $16,6 \%$ & $18,6 \%$ & $7,1 \%$ & $23.8 \%$ & $15,4 \%$ & $17,0 \%$ & $16,7 \%$ \\
\hline \multirow{2}{*}{ Retired } & $n$ & $x 2$ & 8 & 1 & 1 & 3 & 13 & 58 \\
\hline & 5 & $21,2 \%$ & $6.2 \%$ & $2,4 \%$ & $4.8 \%$ & $23,1 \%$ & $13.8 \%$ & $12.9 \%$ \\
\hline \multirow{2}{*}{ Student } & n & 3 & 15 & 18 & 1 & 2 & 10 & 49 \\
\hline & 5 & $20 \%$ & $11,6 \%$ & $42,9 \%$ & $4.8 \%$ & $15,4 \%$ & $10,6 \mathrm{~m}$ & $10,9 \%$ \\
\hline \multirow{2}{*}{ Tradesman } & $n$ & 13 & 10 & 4 & 4 & 2 & 3 & 36 \\
\hline & 5 & $8.6 \%$ & $78 \%$ & $9.5 \%$ & $19,0 \%$ & $15.4 \%$ & $32 \%$ & $8.0 \%$ \\
\hline \multirow{2}{*}{ Cria Servamt } & $\mathrm{n}$ & 3 & 4 & 2 & 0 & 0 & 3 & 12 \\
\hline & $\%$ & $20 \%$ & $3.1 \%$ & $4.8 \%$ & $.0 \%$ & $0 \%$ & $3.2 \%$ & $2,7 \%$ \\
\hline \multirow{2}{*}{ Heatth Omcial } & $n$ & 3 & 2 & 0 & 0 & 0 & 1 & 6 \\
\hline & $\%$ & $2.0 \%$ & $1.0 \%$ & $.0 \%$ & $.0 \%$ & $0 \%$ & $1,1 \%$ & $1,3 \%$ \\
\hline \multirow{2}{*}{ Hairdresser } & $n$ & 0 & 4 & 1 & 0 & 0 & 1 & 6 \\
\hline & 8 & $.0 \%$ & $3.1 \%$ & $2,4 \%$ & $.0 \%$ & $.0 \%$ & $1,1 \%$ & $1,3 \%$ \\
\hline \multirow{2}{*}{ other } & $n$ & 20 & 8 & 0 & 1 & 0 & 9 & 38 \\
\hline & 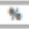 & $13,2 \mathrm{k}$ & $6.2 \%$ & $.0 \%$ & $4.9 \%$ & $.0 \%$ & $9.6 \%$ & $8.4 \%$ \\
\hline \multirow{2}{*}{ Total } & n & 151 & 129 & 42 & 21 & 13 & 94 & 450 \\
\hline & 8 & $100.0 \%$ & $100.0 \%$ & $100.0 \%$ & $100.0 \%$ & $100.0 \%$ & $100.0 \%$ & $100,0 \%$ \\
\hline & & 0,000 & 0,000 & 0,000 & 0,012 & 0,873 & 0,000 & 0,0 \\
\hline
\end{tabular}

\section{DISCUSSION}

Most of the skin diseases have characteristic features according to their localizations on the body. There are studies of the dermatoses localized on head, neck ${ }^{10}$, scalp $^{11}$, ear and nose. in the literature. Although HFD are common in dermatology practice, the studies about this subject are interestingly in small numbers ${ }^{1,2,8,10,12-16}$. These studies have been conducted in different subpopulations of a given society such as children, elderly, patients with systemic diseases like diabetes mellitus, atherosclerosis and immunosuppresion and some occupational groups. ${ }^{7,9,11,13,16}$

In our study we examined 450 patients with only HD and with only FD and with both HFD. When the genders are considered, FD were more prevalent in men and HD were more frequent in women. It may be due to various causes such as greater population of working men and inadequate foot care habits of men in our society.

In the years between 1997 and 1998, 16 European countries have done a multi-centered study called Achilles project on 90.000 patients to elucidate the predisposing factors and the frequency of FD. Burzykowski et al. found $61.3 \%$ of the patients with FD in their study. $41.7 \%$ of the patients were having non fungal FD (mostly orthopedic conditions, corns, psoriasis, eczema, warts) and $40.6 \%$ of the patients were having fungal FD (mostly onychomycosis and tinea pedis). Fungal diseases were common in men while non fungal diseases were common in women. The prevalence of dermatoses was increasing with advancing age. In our study on foot localization we found that fungal infections (54\%) being the most frequent and corns (13\%) and eczema (11\%) respectively. Tinea pedis was seen the most both among men (67\%) and women (54\%). The high prevalence of tinea pedis 
in our country may be due to perform an ablution as a religious ritual behavior and to leave the feet undried after washing. There may be also some other predisposing factors such as obesity, vascular diseases, ostheoarticular pathologies and sportive activities but we did not ask these factors. ${ }^{17}$

Roseeuw et al. found the proportion of patients with FD visiting a dermatologist was high $(58 \%)$ and the prevalence of the FD increased with advancing age and more common in men. The most frequent FD was fungal infections especially onychomycosis (23\%) and tinea pedis (22\%). The prevalence of FD was increasing in advancing ages except warts. Warts were the most common dermatose found in children. In our study we found tinea pedis (33\%) as the most frequent FD and corns (13\%), eczema (11\%) and warts (5\%) were following tinea pedis. Corns were the second most frequent dermatose in our study and we thought that it was due to improper footwear which is common and orthopedic deformities and failure of its management. Warts were also the most frequent dermatoses in children (44\%) in our study. ${ }^{18}$

Cheng et al. found the prevalence of any FD in 1014 patient of their study as $50.7 \%$. They saw $53.1 \%$ of the FD were fungal infections mostly plantar and interdigital tinea pedis $20.4(\%)$ which is similar to our findings. FD were more common in men and in advancing ages. Eczema (6.4\%), corns $(3.9 \%)$, warts $(3.0 \%)$ and pes planus $(2.6 \%)$ were the other common FD seen in their study ${ }^{19}$.

There are many studies of hand eczema in the literature about clinical types of eczema, occupational relation, and quality of life measurement. ${ }^{1,12,20,21}$

Kavli et al. asked to 14.667 person if they had an allergic hand eczema during the previous 12 months and found out $4.9 \%$ of men and $13.2 \%$ of women had had allergic eczema. Women under 35 years old were having 3 times more common hand eczema than men. ${ }^{12}$ Irritan contact eczema was most frequent in women while allergic contact eczema was most frequent in men. We also found that hand eczema was more frequent in women in our study. It was mostly localized to the dorsum of the hands. One can consider that chemical exposures as soaps and detergents in daily life may be the cause. We could not classify the contact dermatitis because we did not apply the patch tests to our patients.

Kavli et al. reported that women with house work as their main occupation had a somewhat as higher frequency of HD than employed women. HD was 4-5 times more frequent in both sexes in less educated compared to well educated individuals, and more frequent in men and women with physically active jobs ${ }^{21}$. In our study we found hand dermatitis was more common in housewifes (40\%).

\section{References}

1. Agner T, Andersen KE, Brandao FM, Bruynzeel DP, Bruze M, Frosh P, et al. 2008. Hand eczema severity and quality of life: a cross-sectional, multicentre study of hand eczema patients. Contact Dermatitis 59(1):43-7

2. Katsambas A, Abeck D, Haneke E, Van de Kerkhof P, Burkowski T, Molenberg G, Marynissen G. 2005. The effects of foot disease on quality of life: results of Achilles Project. J Eur Acad Dermatol Venerol 19(2):191-5 3. Satapathy SK, Bernstein D.Dermatologic disorders and the liver. 2011. Clin Liver Dis 15(1):165-82

4. Serrao R, Zirwas M, English JC. 2007. Palmar erythema. Am J Clin Dermatol 8(6):347-56

5. De Souza CA, Santos Ada C, Santos Lda C, Carneiro AL. 2009. Hereditary tylosis syndrome and esophagus cancer.

An Bras Dermatol 84(5):527-9

6. Turchin I, Barankin B. Dermacase. 2005. Essential or primary hyperhidrosis. Can Fam Physician 51(503):505-6 7. Smith HR, Armstrong DK, Wakelin SH, Rycroft RJ, White IR, McFadden JP. 2000. Descriptive epidemiology of hand dermatitis at the St John's contact dermatitis clinic 1983-97. Br J Dermatol 142(2):284-7.

8. Meding B. Epidemiology of hand eczema in an industral city. 1990. Acta Derm Venerol Supp153:1-43

9. Carrascosa JM, Benvenuti F, Rodriquez C, Ferrandiz C. 2009. Characteristics of patients with hand dermatitis referred to the contact dermatitis unit of a tertiary hospital and impact of patch testing on diagnosis. Actas

Dermosifiliogr 100(6):493-8.

10. Rana RS, Moonis G. Head and neck infection and inflammation. 2011. Radiol Clin North Am 49(1):165-82 11. Hillen U, Grabbe S, Uter W. 2007. Patch test results in patients with scalp dermatitis: analysis of data of the information network of departments of dermatology. Contact Dermatitis 56(2):87-93

12. Kavli G, Førde OH. 1984. Hand dermatoses in Troms $\varnothing$.

Contact Dermatitis 10(3):174-7

13. Van Haalen FM, Brugging SC, Gussekloo J, Assendelft WJ, Eekhof JA. 2009. Warts in primary schoolchildren: prevalence and relation with enviromental factors. Br J Dermatol 161(1):148-52

14. Leggat PA, Smith DR. 2006. Prevalence of hand dermatoses related to latex exposure amongst dentists in Queensland, Australia. Int Dent J 56(3):154-8

15. Jayaprakash P, Bhansali S, Bhansali A, Dutta P, Anantharaman R. 2009. Magnitude of foot problems in diabetes in the developing world: a study of 1044 patients. Diabet Med 26(9):939-42

16. Chaiwanichsiri D, Janchai S, Tantisiriwat N. 2009. Foot disorders and falls in older persons. Gerontology 55(3):296-302

17. Burzykowski T, Molenberghs G, Abeck D, Haneke E, Har R, Katsambas A, et al. 2003. High prevalence of foot diseases in Europe: results of the Achilles Project. Mycoses 46(11-12):496-505

18. Roseeuw D. 1999. Achilles foot screening project: preliminary results of patients screened by dermatologists. $\mathbf{J}$ Eur Acad Dermatol Venereol 12:6-9

19. Cheng S, Chong L. 2002. A prospective epidemiological 
study on tinea pedis and onychomycosis in Hong Kong. Chin Med J 115(6):860-5.

20. Kedrowski DA, Warshaw EM. 2008. Hand dermatitis: a review of clinical features, diagnosis, and management.
Dermatol Nurs 20(1):17-25

21. Kavli G, Førde OH. 1984. Hand dermatoses and sickleave: relationship between educational level, physical activity at work and housework as main occupation. Contact Dermatitis 11(2):70-3 


\section{Author Information}

Gulsen Tukenmez Demirci, MD

specialist, Dermatology Department, Sisli Etfal Training and Research Hospital

\section{Ilknur K?vanc Altunay, MD}

Assoc. Prof, Dermatology Department, Sisli Etfal Training and Research Hospital

\section{Bilge Ates, MD}

resident, Dermatology Department, Sisli Etfal Training and Research Hospital

\section{Gonca Gokdemir, MD}

Assoc. Prof „, Dermatology Department, Istanbul Okmeydanı Training and Research Hospital 